\title{
Isolated large renal hydatid cyst treated by laparoscopic nephrectomy
}

\author{
Veda Murthy Reddy Pogula ${ }^{1}$, Ershad Hussain Galeti ${ }^{1}$, Bhargava Reddy Kanchiv ${ }^{1}$, Ifrah Ahmad ${ }^{1}$, Ayesha Galeti ${ }^{1}$
}

Cite this article: Pogula V, Galeti E, Kanchiv B, Ahmad I, Galeti A: Isolated large renal hydatid cyst treated by laparoscopic nephrectomy. Ann Urol Oncol 2021; 4(1): 5-9. https://doi. org/10.32948/auo.2021.08.16

\begin{abstract}
Hydatid disease is caused by Echinococcus granulosus, which causes rare isolated presentation in the kidneys, and is estimated to be about $2-4 \%$ of all cases. We herein present a case of a 45-year-old symptomatic male patient with a large primary hydatid cyst in the left kidney that was treated successfully by laparoscopic left nephrectomy.
\end{abstract}

Key words Renal hydatid disease, laparoscopic nephrectomy, hydatiduria, urogenital

1. Narayana medical college, Nellore, Andhra Pradesh, India.

Correspondence: Ershad hussain galeti (Department of urology, Narayana medical college, nellore, 524003, Andhra pradesh, India; Email: dr.ershadhussain@gmail.com). 


\section{Introduction}

Kidney involvement in Echinococcosis caused by tapeworm is extremely rare and it comprises of only $2-3 \%$ of all cases [1]. It is caused in humans by larval stage of Echinococcus granulosus complex, E. multilocularis, E. vogeli, or E. granulosus. Most patients with primary involvement of kidney remain asymptomatic for many years [2]. The patients may be asymptomatic or present with flank pain, hematuria, and hydaturia. Surgery is the mainstay of treatment, and options include cyst deroofing, partial nephrectomy, and total nephrectomy. We share our experience of laparoscopic management of a patient with large hydatid cyst of the left kidney.

\section{Case report}

A 45-year-old man presented to our department with chronic left loin pain, which was non-radiating with intermittent fever for the past six months. Clinical examination revealed a smooth, non-tender mass in the left flank. Abdominal ultrasonography demonstrated a multicystic lesion in the left kidney. Computed tomography (CECT) of the abdomen showed a heterogeneously hypodense multicystic lesion of $17 \mathrm{x} 9 \mathrm{~cm}$ with calcified thick and irregular walls, with small multiple daughter cysts within the lesion, which is a characteristic of hydatid disease (Figure 1a \& 1b). A complete blood count revealed mild eosinophilia $(10 \%)$ and raised erythrocyte sedimentation rate $(26 \mathrm{~mm} /$ hour $)$ Preoperatively. patient was given oral albendazole $(400 \mathrm{mg})$ twice daily for two weeks. After an informed consent, a laparoscopic left-sided nephrectomy was planned. With the patient under general anaesthesia and in the right flank position and with three ports, the kidney was mobilized outside gerota's fascia without any spillage. The renal artery, renal vein and ureter were dissected, clipped and cut. A small left iliac fossa incision was made, and the specimen delivered out within the specimen bag without spillage and drain placed (Figure 2a). The cut surface of the mass showed a well-developed multilocular cyst involving the majority of renal parenchyma and lined by a membrane detached from the surrounding walls containing multiple small daughter cysts (Figure 2b).The patient was started on liquid orals the next day andwas discharged on the fifth postoperative day. Histopathological examination described hyalinized cyst wall with lamellated membrane and brood capsules adjacent to thickened renal parenchyma along with few tubules showing thyroidisation and chronic mononuclear inflammatory cells and excluded malignancy (Figure 3a \& 3b). A course of albendazole $400 \mathrm{mg}$ twice daily was given for one month to reduce relapse possibilities.

\section{Discussion}

Hydatid disease is a zoonotic disease caused by the parasite Echinococcus granulosus. Eggs are excreted in the definitive host's faeces (usually dogs and sheep). Humans commonly get infected through contact with the definitive host or ingesting contaminated soil, water and vegetables. These eggs transform to larvae in the human's digestive system and migrate into the small bowel wall and into the mesenteric circulation, where it gets filtered by the liver, which is why hepatic infection is so common. The second most common infiltration site is the lung, which is involved in $15 \%$ of the cases [3]. Kidney involvement represents $2-4 \%$ of all cases and is rare compared to that of the liver and lung, even rarer as an isolated site of infection [1]. The current case did not have any history of contacting animals. Thus, probably infestation may have occurred through contaminated foods.

Although it has no specific clinical presentation, the renal hydatid cyst has a characteristic appearance on imaging, surgical cutsurface and histopathological examination. The triad of relevant clinical history, imaging tools, and laboratory investigations provides a reliable preoperative diagnosis in about half of the cases. Despite serological tests and having significant imaging techniques, the diagnosis of renal cyst hydatid cannot be made in $1 / 3$ of the patients before the operation [4]. In our case, renal hydatid cyst was diagnosed before the surgery.

On imaging studies in the current case, a well-demarcated multilocular renal cyst was seen. The detached germinative membrane had a two-layered membrane, with the cyst wall, which is referred to as "double-contour" appearance. This appearance has been previously described as "water-lilysign". Echinococcal cystic lesions have been classified by the WHO into five grades (Table 1). Based on this WHO classification, imaging findings of
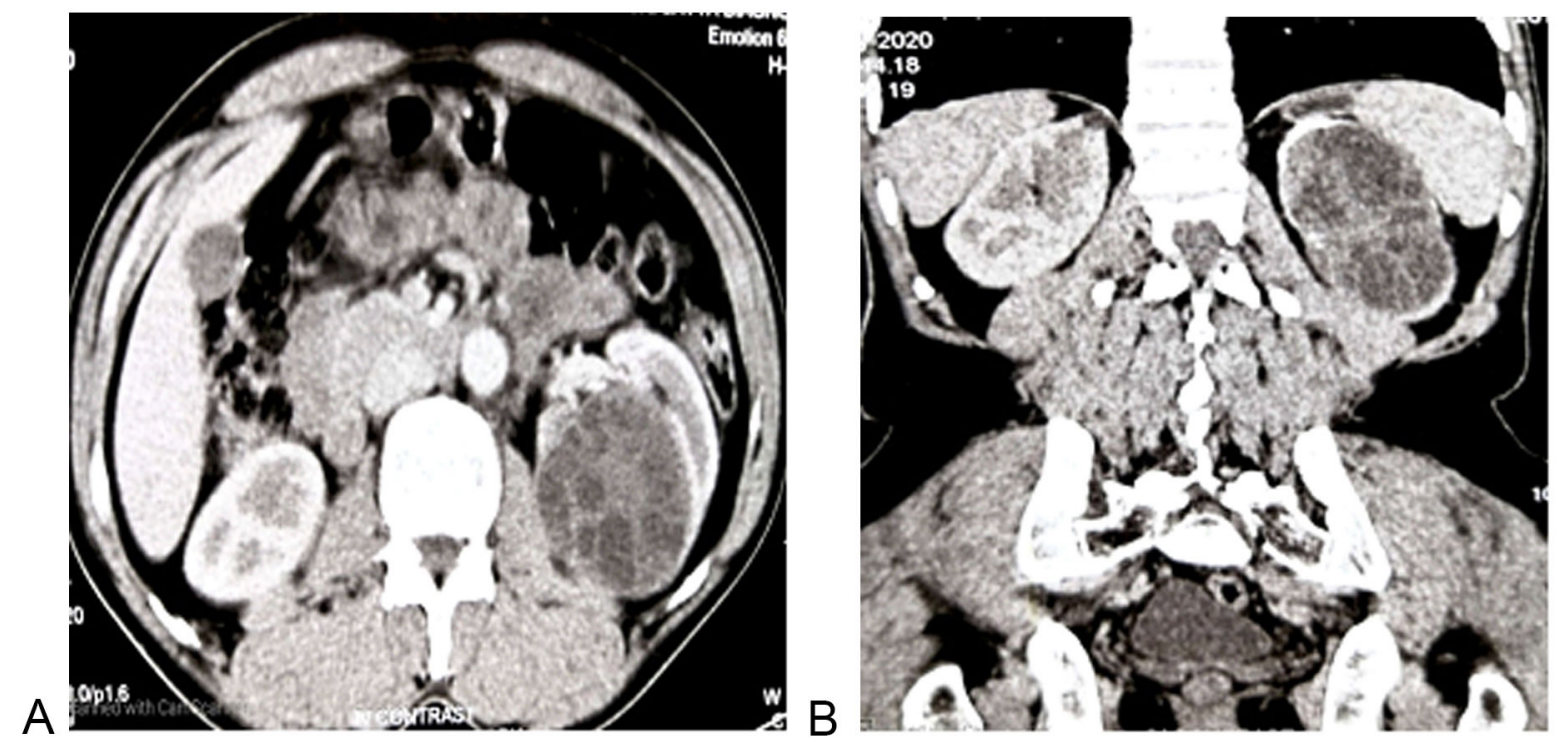

Figure 1 : Abdominal CECT. a: Axial view showing a multi-loculated hydatid cyst involving the left kidney; b: Coronal view showing a multiloculated Hydatid cyst involving the majority of the left kidney. 
A


Figure 2 : Nephrectomy specimen. a: Laparoscopic left nephrectomy gross specimen; b: Left Nephrectomy specimen with multiple daughter cysts.

the current case refers to Grade $3 b$ hydatid cyst [5,6]. Symptoms and signs of the hydatid cyst disease depend on the involved organ and the secondary spread. The most common clinical finding is a palpable mass. Patients with renal hydatid cystic disease usually present vague pain in the flank region [7]. The cyst's may rupture into the collecting system causing hydatiduria, which is a pathognomonic sign of renal hydatidosis. It is present in only 15 $20 \%$ of the cases. Eosinophilia is noted in $50 \%$ of cases. Pre and post-operatively use of albendazole decreases the cyst wall tension thus reducing the risk of spillage during surgery and prevent the chance of anaphylaxis [8]. Surgery is the treatment option, either through an open or laparoscopic approach [9]. The cyst should be removed without rupture to avoid the spread of the disease, and the kidney sparing cyst removal is performed by cystectomy and pericystectomy when possible. Superficial renal hydatid cysts that do not involve the renal parenchyma can be treated by cystectomy or pericystectomy. However, in cases in which the cyst extends deep into the renal parenchyma, partial nephrectomy can be performed. However, an attempt must be made to preserve as much of the parenchyma as possible [10]. These nephron-sparing
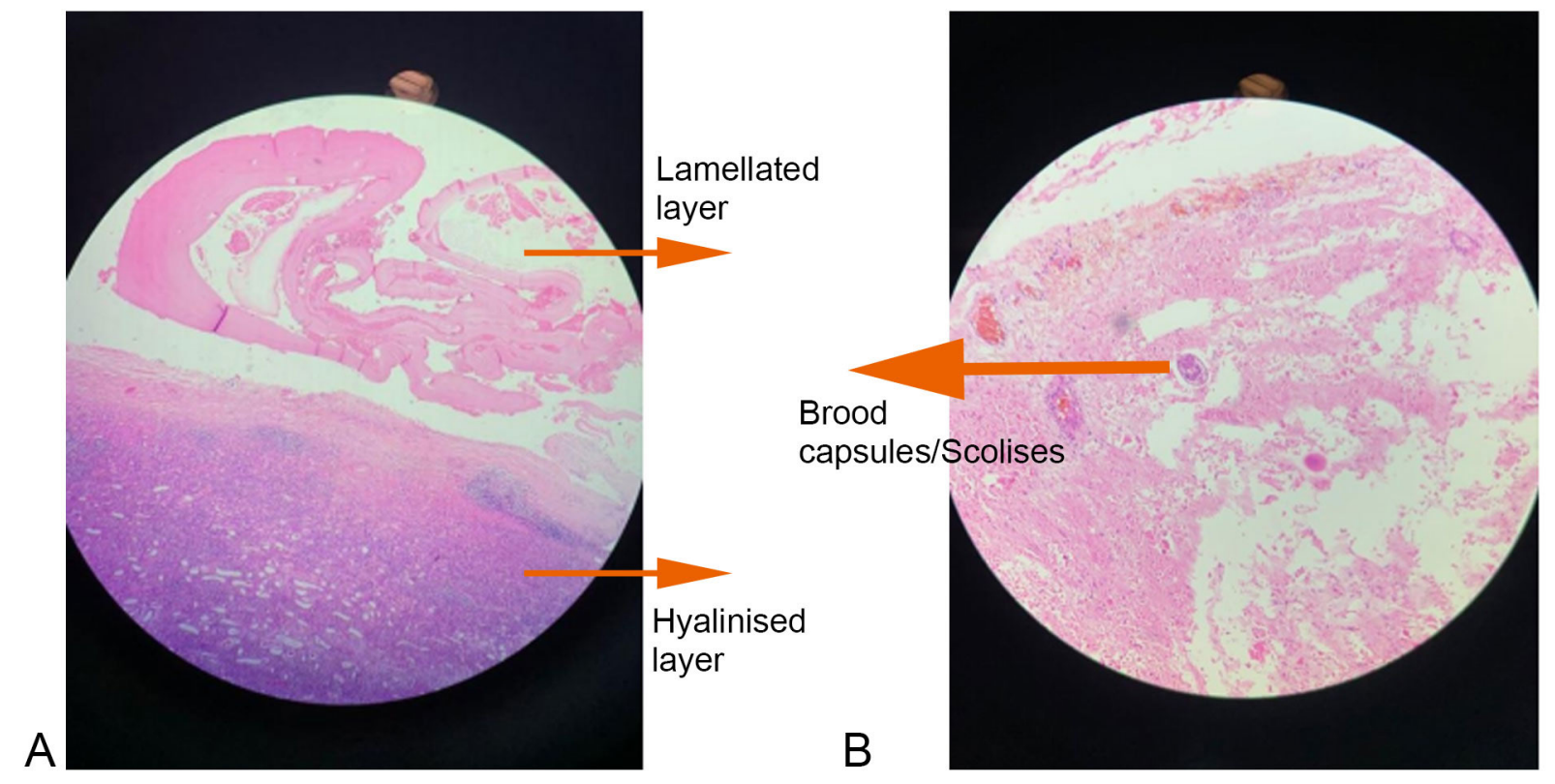

Figure 3a : Hematoxylin and Eosin stain x10 magnification of cyst wall. Figure 3b : Hematoxylin and Eosin stain x40 magnification of cyst wall. 
Table 1: Classification of imaging results based on the WHO classification of cystic echinococcosis.

\begin{tabular}{|c|c|}
\hline WHO Classification & Imaging characteristics \\
\hline CL & $\begin{array}{l}\text { Univesicular, cystic lesion with uniform echoes, clear boundary, thin visible wall. If it is a hydatid } \\
\text { cyst, it is active. }\end{array}$ \\
\hline CE1 & $\begin{array}{l}\text { Univesicular anechoic cyst. Presence of hydatid sand, snow flake sign and double wall sign. The hy- } \\
\text { datid is active }\end{array}$ \\
\hline CE2 & $\begin{array}{l}\text { Multivesicular, multiseptated cysts; cysts septations produce "wheel-like"structures, and presence of } \\
\text { daughter cysts is indicated by "rosette-like" or "honeycomb-like" structures. }\end{array}$ \\
\hline CE3a & $\begin{array}{l}\text { Detachment of laminated membrane from the cyst wall visible as "big snake sign" or as "wa- } \\
\text { ter-lily sign". The hydatid status is transitional. }\end{array}$ \\
\hline CE3b & $\begin{array}{l}\text { Intracystic shadow of the daughter vesicles and solid septation, manifested as complex cyst shadow. } \\
\text { The hydatid is dying. }\end{array}$ \\
\hline CE4 & Heterogenous hypoechoic or hyperechoic contents ecurrence of hydatid disease. \\
\hline CE5 & Intracystic solid degeneration and calcification of the cystic wall. The hydatid is inactive \\
\hline
\end{tabular}

*CE- Cystic Echinococcosis

surgeries are possible in $75 \%$ of the cases, and total nephrectomy should be reserved only for cases in which the entire kidney is occupied by the cyst [2]. Nephrectomy is usually needed when the hydatid cyst invades a major renal part or in cases of hydatiduria. The other problem faced in laparoscopic treatment of hydatid cysts is the difficulty in evacuating the articulate contents of the cyst, the daughter cysts, and laminated membranes. Failure to remove all viable protoscolices in the initial operation may result in local recurrence [11]. There are few reports of laparoscopic removal of renal hydatid but chances of cyst rupture, dissemination, and incomplete removal of the hydatid cyst are quite high [12]. In our case, we considered feasible a laparoscopic left-sided nephrectomy because the hydatid cyst invaded a majority of the renal parenchyma. Ozden et al. also applied pericystectomy with a laparo- scopic retroperitoneal approach to their patient to avoid risk of intraperitoneal contamination [13]. We did not apply peri-cystectomy in our patient. The postoperative period was uncomplicated, and the patient received albendazole for four weeks. After a six-month follow-up, the patient was asymptomatic. The laparoscopic approach can either be retroperitoneal [14] or trans-peritoneal [15]. In our opinion, the laparoscopic retroperitoneal approach may be preferred in cases without giant hydatid cysts. However, the laparoscopic transperitoneal approach will be more appropriate when it is large or giant-sized, as in our case. Although there is a risk of intraperitoneal contamination, the transperitoneal approach provides a better workspace for the operating surgeon if carefully done.

We conclude by stating that laparoscopic renal hydatid cyst surgery may be performed with minimal morbidity, minimal intraoperative blood loss, without intraperitoneal spillage, improved cosmetic results, shorter hospital stay, and an early return to work, making it the gold standard in the treatment of renal hydatid disease.

\section{Acknowledgements}

NIL

\section{Ethical policy}

Approval was taken from institutional ethical committee. The study was performed in accordance with the Declaration of Helsinki. Patients gave their informed consent for their participation.

\section{Author contributions}

All authors have equally contributed to the manuscript.

\section{Competing interests}

NIL

\section{Funding}

NIL

\section{References}

1. Narang S, Handa U, Nanda A, Bansal R, Nahar R, Sood S. Primary intravitreal hydatid cyst: diagnosis on cytological examination. Annals of Tropical Medicine \& Parasitology 2006, 100(4): 371-374. https://doi.org/10.1179/136485906x91495.

2. Zmerli S, Ayed M, Horchani A, Chami I, El Ouakdi M, Ben Slama MR. Hydatid cyst of the kidney: diagnosis and treatment. World J Surg 2001, 25: 68-74.

3. Bandyopadhyay A, Khatua S, Das S, Bose K, Konar K. A rare case of primary renal hydatid cyst presenting with hydatiduria. J Parasit Dis 2013, 39(3): 577-580. https://doi.org/10.1007/s12639-013-0362-x.

4. Angulo JC, Sanchez-Chapado M, Diego A, Escribano J, Tamayo JC, Martin L. Renal echinococcosis: Clinical study of 34 cases. J Urol 1997, 157: 787-794.

5. Brunetti E, Kern P, Vuitton DA. Expert consensus for the diagnosis and treatment of cystic and alveolar echinococcosis in humans. ActaTropica 2010, 114(1): 1-16. https://doi.org/10.1016/ j.actatropica.2009.11.001.

6. Stojkovic M, Rosenberger K, Kauczor HU, Junghanss T, Hosch W. Diagnosing and Staging of Cystic Echinococcosis: How Do CT and MRI Perform in Comparison to Ultrasound? PLoS Neglected 
Tropical Diseases 2012, 6(10): e1880. https://doi.org/10.1371/journal. pntd.0001880.

7. Bhat, GS. Isolated Renal Hydatid Cyst Masquerading as Cystic Renal Cell Carcinoma: A Case Report. J Clin Diagn Res 2015, 5760. https://doi.org/10.7860/jcdr/2015/11488.5730.

8. A Cushieri, RJC Steele, and AR Moosa. Treatment of Hydatid Cyst, Essential Surgical Practice, Arnold, 4th edition.

9. Shahait M, Saoud R, El Hajj A. Laparoscopic treatment of giant renal cystic echinococcosis. Int J Infect Dis 2016, 42: 58-60. https:// doi.org/10.1016/j.ijid.2015.11.016.

10. Tryfonas GJ, Avtzoglou PP, Chaidos C, Zioutis J, Gavopoulos S, Limas C. Renal hydatid disease: diagnosis and treatment. J Pediatr Surg 1993, 28: 228-231.

11. Durakbasa CU, Tireli GA, Sehiralti V, Sander S, Tosyali AN, Mutus M. An audit on pediatric hydatid disease of uncommon localization: Incidence,diagnosis, surgical approach, and outcome. J Pediatr Surg 2006, 41: 1457-1463.

12. A Basiri, M Nadjafi-Semnani, A Nooralizadeh. Laparoscopic partial nephrectomy for isolated renal hydatid disease. J Endourol 2006, 20(1): 24-26.

13. Ozden E, Bostanci Y, Mercimek MN, Yakupoglu YK, Yilmaz AF, Sarıkaya S. Renal hydatid cyst treatment: Retroperitoneo-scopic “closed cyst" pericystectomy. Int J Urol 2011, 18: 237-239.

14. Rabii R, Mezzour MH, Essaki H, Fekak H, Joual A, Meziane F. Laparoscopic Treatment for Renal Hydatid Cyst. J Endourol 2006, 20(3): 199-201. https://doi.org/10.1089/end.2006.20.199.

15. Desai MR, Shah KJ, Ganpule AP. Isolated renal hydatid cyst managed by laparoscopic transperitoneal nephrectomy. Indian J Urol 2009, 25(4): 531. https://doi.org/10.4103/0970-1591.57925. 